\title{
Editorial
}

\section{Fiber Optic Sensors: Perspectives and Challenges}

Marco Pisco ${ }^{1, *}$ and Agostino Iadicicco, ${ }^{2, *}$

\author{
${ }^{1}$ University of Sannio, Optoelectronic Division - Engineering Department Palazzo Bosco Lucarelli - Corso Garibaldi \\ 107, 82100 - Benevento, Italy \\ ${ }^{2}$ Department of Engineering, University of Napoli "Parthenope" Centro Direzionale - Isola C4 - 80143, Napoli, Italy
}

Fiber optics reached the present worldwide diffusion thanks to several knowledge and technology breakthroughs. Guiding of light by refraction, the principle that made first fiber optics possible, was demonstrated by Jean-Daniel Colladon, a 38-year-old Swiss professor at University of Geneva, in 1841. He wanted to show the fluid flow through various holes of a tank and, to help audience seeing, he collected sunlight through a tube. The light was focused through the water tank and was made to incident on the edge of the jet at a glancing angle. Instead of traveling in a straight line, the light followed the curvature of the water flow by total internal reflection until the water jet broke up. Right after, Colladon demonstrated light guiding in water jets through a number of public performances to the urban intelligentsia of Paris.

John Tyndall included a demonstration of it in his public lectures at the Royal Institution in London, about 12 years later. Tyndall's experiments, although featured by educational purposes, had the merit to stimulate research efforts, in a broader scientific audience, to guide light with more control than could be achieved in a jet of water.

Attempts to exploit total internal reflection in the medical industry were also successively performed. Bent rods of glass were used to illuminate internal organs by Roth and Reuss at Vienna (1888). In the following years, the possibility to guide light in glass rods was variously proposed for lighting and imaging applications.

Nevertheless, the fiber optic technology made significant steps forward only in the second half the nineteen century. In these years a cladding, covering the "core" of the fibers, was proposed to improve the transmission properties. Moreover, in the 1950s there was the first all-glass fibrescope for medical applications (H. H. Hopkins and N. S. Kapany (1954)).

In the 1966, C. K. Kao demonstrated that losses in dielectric media were mostly caused by absorption and scattering

\footnotetext{
*Address correspondence to these authors University of Sannio, Optoelectronic Division - Engineering Department Palazzo Bosco Lucarelli - Corso Garibaldi 107, 82100 - Benevento, Italy; Tel: +390824305810; Fax:+390824305846; E-mail: pisco@unisannio.it.

University of Naples "Parthenope", Department of Engineering, Centro Direzionale Isola C4, 80143, Napoli, Italy; Tel: +390815476718;

Fax:+390815476777; E-mail: iadicicco@uniparthenope.it
}

and envisioned that fibers with glass of higher purity could be a new form of communication medium.

In the following years, the fiber optic technology experienced a considerable grown pushed by the research efforts to improve the light transportation capability. The attenuation losses strongly decreased using different core dopants and fiber fabrication approaches.

In parallel with communication developments, significant efforts were carried out by a number of different research groups around the world to exploit them also in sensing applications. The inherent advantages of fiber optic sensors, which include their ability to be lightweight, very small in size, passive, low power, resistant to electromagnetic interference, high sensitive, with wide bandwidth and environmental ruggedness, were heavily used to offset their major disadvantages of high cost and unfamiliarity to the end user. Such properties have made optical fiber probes suitable to sense various physical, chemical, and biological parameters. As a result, commercializing optical fiber sensors become great interest to many companies.

Despite the communication applications where the light guided in fiber optic needs to be kept as more as insensitive to external or environmental parameters, in sensing applications the light needs to interact directly or indirectly with measurand. Any measurand acting on one or more light parameters such as phase, amplitude, wavelength and so on can be detected. Based on this simple consideration, in relatively few years, the fiber optic sensing potentialities have been widely improved by the advance of various technological platform and/or physical phenomena such as fiber gratings, fiber interferometers, Brillouin scattering, surface plasmon resonance, etc.

Besides, new optical fiber design in terms of shape and/or materials also plays a fundamental role in looking for novel approaches that could give rise to innovative control of optical waves. A large set of special optical fibers were proposed and demonstrated in the last decades, ranging from plastic optical fibers, Bragg fibers to specific fibers such as double core optical fibers and D shaped fibers and many others constructed for specific applications. Furthermore, new mechanisms capable to control the fiber guided light or new effects to properly modify the optical patch are investi- 
gated with the aim to manage the interaction of the light and measurand.

In this special issue, we focused the attention exclusively on a limited number of invited review articles. Inevitably many important aspects are omitted but we believe that they however well represent the current state of the art and current trends in the studies and applications of the basic topics of optical fiber sensors.

Specifically, we were pleased to start this special issue with an excellent review paper authored by Brian Culshaw for his efforts in fiber optic sensors development. Here, he presents a brief overview of the principles which have evolved and the achievements which have accrued over almost half a century of fibre optic sensors.

The following papers were focused on new phenomena and sensing mechanisms involved in design of novel optical fiber sensors.

Gilberto Brambilla was invited to submit a wide review of optical microfiber and nanofibers (MNF) based sensors reported to-date. Sensors are divided according to their morphology and measurand. This review reports several NMF based sensors including sensors configurations driven by powerful industries such as automotive, biomedical and defense, but also novel sensors for measuring refractive index, bio-chemical, temperature, current, displacement, bend, surface, acceleration, force, rotation, acoustic, electric field and magnetic field.

Banshi D. Gupta was invited to present a review paper as a rigorous and organized literature for the understanding of the basics, utility and trends of fiber optic plasmonic sensors. They reviewed various fiber optic sensors utilizing both the propagating and localized surface plasmon resonance techniques. They clearly highlight that utilization of optical fibers in plasmon based sensing has provided several advantages in sensing of various physical, chemical and biochemical parameters.

We also invited Francisco J. Arregui to write a review of nanostructured materials employed in the fabrication of optical fiber sensors in the last years. The continuous advances in nanofabrication techniques have enabled to manipulate the matter precisely producing well defined nanostructurated coatings or repetitive patterns at nanoscale level. The interactions of light with these nano-organized materials or patterns at the nanoscale level enable to observe interesting phenomena, such as interferometry, fluorescence, absorbance, resonances and many others which can be exploited in the fabrication of sensing devices.

An exhaustive invited review about the latest achievements obtained with bare and coated tilted fiber Bragg gratings used for high-resolution refractometry and biochemical sensing through surface Plasmon wave generation was provided by Christophe Caucheteur et al. from University of Mons (Belgium).

Among different sensing configuration, the distributed optical fiber sensors are based on the scattering processes that originate from the interaction between light and matter. The three different scattering processes that may take place in a fiber are named Rayleigh, Raman and Brillouin scattering. Luca Palmieri was invited to write a review paper focused on Rayleigh-based distributed optical fiber sensors.

Gianluca Gagliardi was invited to write a review paper on fiber-optic resonators for their capability to act as ideal mechanical probes and chemical sensors.

Finally, the different sensing mechanisms above discussed make the optical fiber sensors attractive for several applications including high vibration, extreme heat, noisy, wet, corrosive or explosive environments. As example, Wojtek J. Bock was invited to submit a review paper on fiberoptic sensors for the detection of trace vapor explosive materials. The rapid evolution of laser-induced breakdown spectroscopy and Raman spectroscopy from benchtop instruments to field-deployable and portable units is emphasized as these techniques are widely used for detection of trace explosives and strongly related to fiber-optic sensing.

We hope that the readers will find this special issue a useful guide and introduction to the many exciting ways that optical fibers are being applied to a variety of problems in sensing.

(C) Pisco and Iadicicco; Licensee Bentham Open.

This is an open access article licensed under the terms of the Creative Commons Attribution Non-Commercial License (http://creativecommons.org/licenses/by-nc/3.0/) which permits unrestricted, non-commercial use, distribution and reproduction in any medium, provided the work is properly cited. 